\title{
Ocular hypertension in myopia: analysis of contrast sensitivity
}

\author{
Arrico L*, Migliorini R, Quercia A, Bianchini D and Nebbioso M \\ Department of Sense Organs, University of Rome “La Sapienza”, Italy
}

\begin{abstract}
Purpose: we evaluated the evolution of contrast sensitivity reduction in patients affected by ocular hypertension and glaucoma, with low to moderate myopia. We also evaluated the relationship between contrast sensitivity and mean deviation of visual field.

Material and methods: 158 patients (316 eyes), aged between 38 and 57 years old, were enrolled and divided into 4 groups: emmetropes, myopes, myopes with ocular hypertension (IOP $\geq 21 \pm 2 \mathrm{mmHg}$ ), myopes with glaucoma. All patients underwent anamnestic and complete eye evaluation, tonometric curves with Goldmann's applanation tonometer, cup/disc ratio evaluation, gonioscopy by Goldmann's three-mirrors lens, automated perimetry (Humphrey 30-2 full-threshold test) and contrast sensitivity evaluation by Pelli-Robson charts. A contrast sensitivity under 1,8 Logarithm of the Minimum Angle of Resolution (LogMAR) was considered abnormal.

Results: contrast sensitivity was reduced in the group of myopes with ocular hypertension (1,788 LogMAR) and in the group of myopes with glaucoma (1,743 $\operatorname{LogMAR})$, while it was preserved in the group of myopes (2,069 LogMAR) and in the group of emmetropes (1,990 LogMAR). We also found a strong correlation between contrast sensitivity reduction and mean deviation of visual fields in myopes with glaucoma (coefficient relation $=0.86$ ) and in myopes with ocular hypertension (coefficient relation $=0.78$ ).
\end{abstract}

Conclusions: the contrast sensitivity assessment performed by the Pelli-Robson test should be performed in all patients with middle-grade myopia, ocular hypertension and optic disc suspected for glaucoma, as it may be useful in the early diagnosis of the disease.

\section{Introduction}

Contrast can be defined as the ability of the eye to discriminate differences in luminance between the stimulus and the background.

The sensitivity to contrast is represented by the inverse of the minimal contrast necessary to make an object visible; the lower the contrast the greater the sensitivity, and the other way around.

Contrast sensitivity is a fundamental aspect of vision together with visual acuity: the latter defines the smallest spatial detail that the subject manages to discriminate under optimal conditions, but it only provides information about the size of the stimulus that the eye is capable to perceive; instead, the evaluation of contrast sensitivity provides information not obtainable with only the measurement of visual acuity, as it establishes the minimum difference in luminance that must be present between the stimulus and its background so that the retina is adequately stimulated to perceive the stimulus itself $[1,2]$.

The clinical methods of examining contrast sensitivity (lattices, luminance gradients, variable-contrast optotypic tables and lowcontrast optotypic tables) relate the two parameters on which the ability to distinctly perceive an object depends, namely the different luminance degree of the two adjacent areas and the spatial frequency, which is linked to the size of the object.

The measurement of contrast sensitivity becomes valuable in the diagnosis and follow up of some important eye conditions such as glaucoma. Studies show that contrast sensitivity can be related to data obtained with the visual perimetry, especially with the perimetric damage of the central area and of the optic nerve head $[3,4]$.
Slight loss of vision, both central and peripheral, can be seen in some patients with glaucoma even before visual field defects detectable with standard techniques, via measuring the amount of contrast necessary to distinguish adjacent visual stimuli.

Psychophysical studies confirm that even in the highly myopic eye, in which the cellular elements are elongated due to the larger size of the bulb, there is a reduction in the sensitivity to contrast, both spatial and temporal; visual sensitivity and spatial additions are reduced, implying damage to photoreceptors and postreceptor elements $[5,6]$.

Myopia has always been considered one of the risk factors in glaucoma: the effect of stretching and traction of lamina cribrosa lamellae by scleral tension may play a role in the pathogenesis of glaucoma. Glaucoma and myopia also have a documented hereditary base and may share genes in common.

To date one of the main problems of ophthalmology that is still not completely resolved is the early diagnosis of simple chronic glaucoma. The latter, together with the timely detection of structural damage

${ }^{*}$ Correspondence to: Loredana Arrico, Department of Sense Organs, Glaucoma Center, University of Rome "La Sapienza", Via del Policlinico 155, 00161 Rome, Italy, E-mail: loredana.arrico@uniromal.it

Key words: contrast sensitivity, glaucoma, myopia, ocular hypertension, Pelli Robson test

Received: August 09, 2018; Accepted: August 20, 2018; Published: August 23, 2018 
progression are two objectives of primary importance; since the anatomical damage is irreversible, the therapeutic strategy essentially lies in prevention $[7,8]$.

With these premises, which indicate that myopia and glaucoma affect negatively the sensitivity to contrast, we decided to tackle the study of contrast sensitivity in order to evaluate the changes in myopia of mild and medium grade vs glaucoma and myopia.

The aim of our study was to evaluate whether a reduction in contrast sensitivity can be predictive of the evolution of ocular hypertension (IOP) towards full-blown glaucoma in myopic patients, with ocular hypertension, excellent visual acuity and preserved retinal sensitivity at the computerized perimetry.

\section{Materials and methods}

We enrolled 4 groups of patients (158 patients, 316 eyes): GROUP 1 (EM), 40 healthy patients (80 eyes), emmetropes without ocular diseases or ametropia; GROUP 2 (MY), 38 patients (76 eyes) with myopia, without ocular diseases; GROUP $3(\mathrm{MH}), 38$ patients (76 eyes) with myopia, ocular hypertension and optic nerve head alteration but normal Visual Field Humphrey H 30-2, not under treatment; GROUP 4 (MG), 42 patients (84 eyes) with myopia, affected by primary open-angle glaucoma in tonometric compensation under treatment, followed by the Glaucoma Centre of "Department Testa-Collo U.O.C. B, Università degli Studi di Roma La Sapienza”.

Inclusion criteria were: $4^{\text {th }}$ grade-angle (according to Shaffer); ocular pressure $\geq 21 \mathrm{mmHg}$ (IOP) in the group of patients with myopia and ocular hypertension; myopia between $-0.50 \mathrm{D}$ and $-5 \mathrm{D}$; pachymetry of 500-550 $\mu \mathrm{m}$; visual acuity (VA) between $4 / 10$ and 10/10 expressed in Logarithm of the Minimum Angle of Resolution (logMAR); Visual Field (VF) H30-2 with low defects (stage 0) according to Brusini's Glaucoma staging system (GSS 2 2006) and pathological Glaucoma Hemifield Test.

Exclusion criteria were: cardiovascular diseases and diabetes or systemic treatments; monocular patients; microphthalmos, aniridia and other congenital pathologies; congenital close-angle glaucoma; patients undergone ocular surgery or laser treatments; patients affected by uncontrollable glaucoma, not under treatment or treated with glaucoma surgery; patients with an astigmatism over 1,5 D; anterior segment disorders as cataract, pseudoexfoliative syndrome, uveitis, rubeosis iridis, corneal dystrophies, corneal scars, etc; posterior segment disorders as maculopathy, retinal detachment, diabetic retinopathy, hypertensive retinopathy, etc.

All patients received: anamnestic evaluation and complete eye examination; tonometric curves with Goldmann's applanation tonometer; cup/disc ratio evaluation by biomicroscopy with Volk's lens +90D; gonioscopy by Goldmann's three-mirrors lens; automated perimetry (Humphrey 30-2 full-threshold test); contrast sensitivity evaluation by Pelli-Robson charts, 2 measurement (CSO VISION CHART Mod. CVC03).

The Pelli-Robson tables are composed by a series of 48 letters, divided into 16 groups of 3 letters each, $4.9 \mathrm{~cm}$ high, placed a meter away from the patient, that allow to evaluate the sensitivity to contrast at low spatial frequencies, around at $0.5 / 1$ cycles per degree (c.p.g.); the test can also be administered at a distance of 3 meters to evaluate higher spatial frequencies, around 1.5 / 2 c.p.g.

Within the same triplet the letters have the same contrast, and the contrast of each successive triplet decreases by 0.15 logarithmic units.
The room should be well and uniformly lit, and the monitor should be placed at $1 \mathrm{~m}$ from the patient, orthogonally to his sight.

Using the best spherocylindrical correction for the two eyes, the examination is first performed monocularly and then binocularly: the triplets are presented in succession, starting from the higher contrast and reducing the contrast until the patient misses at least 2 letters of the triplet. To calculate the threshold value, the contrast corresponding to the triplet of the last letter correctly viewed is marked, then 0.05 logarithmic units are subtracted for each wrong letter in the previous screens [1]. The contrast sensitivity is then measured in LogMAR and a contrast sensitivity of less than 1.8 LogMAR is considered as altered [2]. The Pelli-Robson test is technologically a not very advanced test; but its simplicity of execution, low cost, reproducibility and reliability make it the most used test in ophthalmic clinics to date. The only disadvantage is the fact that the results may vary depending on the time given to the patient to recognize the letters close to the threshold value. [9-12]

Statistical analysis was performed using Pearson's linear correlation and linear regression analysis between the visual acuity expressed in LogMAR and the contrast sensitivity measured in the Pelli-Robson test and expressed in LogMAR, then correlating visual acuity and sensitivity to contrast with the average deviation of the visual field.

The comparability of the study groups was instead evaluated by means of ANOVA and t Student test. Differences with $\mathrm{P} \leq 0.05$ were considered significant.

\section{Results}

The groups resulted homogeneous and therefore comparable for all the listed variants; age, sex, refraction and axial length (Tables 1 and 2).

1. GROUP 1: Emmetropes (EM). 18 males, 22 females, mean age $47.3 \pm 9.7$ years, mean IOP $13.8 \pm 1.5 \mathrm{mmHg}$, axial length 24.16 $\mathrm{mm} \pm 1.3$, visual acuity $10 / 10$ natural, MD $0.4563 \mathrm{~dB}$ (sd 0.58 ), CS 2.069 LogMAR (sd 0.16). Correlation CS-MD of $0.3563(\mathrm{P}=0.0012)$, correlation visual acuity-MD of -0.1753 ( $\mathrm{P}=0.1198$, not significant).

2. GROUP 2: Myopes (MY). 21 males, 17 females, mean age 46.07 \pm 8.3 years, mean IOP $13.7 \pm 1.7 \mathrm{mmHg}$, axial length $24.16 \pm 0.9$ $\mathrm{mm}$, mean visual acuity $10 / 10$ with mean spherical equivalent of $-1.6 \pm 1.3$ D, MD $-0.098 \mathrm{~dB}$ (sd 0.92), CS 1.99 LogMAR (sd 0.17). Correlation CS-MD of $0.5171(\mathrm{P}<0.0001)$, correlation visual acuity$\mathrm{MD}$ of -0.02657 ( $\mathrm{P}=0.8298$, not significant $)$.

Table 1. Anagraphical data of patients enrolled

\begin{tabular}{|c|c|c|c|}
\hline Group & N & Sex M/F & Age \\
\hline 1) EM & 40 & $18 / 22$ & $47.3 \pm 9.7$ \\
\hline 2) MY & 38 & $21 / 17$ & $46.07 \pm 8.3$ \\
\hline 3) MH & 38 & $17 / 21$ & $47.5 \pm 7.6$ \\
\hline 4) MG & 42 & $19 / 23$ & $48 \pm 9.6$ \\
\hline
\end{tabular}

1-way ANOVA $\mathrm{P}=0.8$ : age was not statistically significant

$\mathrm{T}$ test $\mathrm{P}=0.24: \mathrm{M} / \mathrm{F}$ ratio was not statistically significant

Table 2. IOP, refractive defects and axial length of patients enrolled

\begin{tabular}{|c|c|c|c|c|c|}
\hline Group & $\mathbf{N}$ & $\begin{array}{c}\text { IOP } \\
(\mathbf{m m H g})\end{array}$ & $\begin{array}{c}\text { Spherical } \\
\text { equivalent (D) }\end{array}$ & $\begin{array}{c}\text { Axial length } \\
\mathbf{( m m )}\end{array}$ & $\begin{array}{c}\text { Correlation } \\
\text { coefficient (r) }\end{array}$ \\
\hline 1) EM & 40 & $13,8 \pm 1.5$ & 0 & $24,16 \pm 1.3$ & - \\
\hline 2) MY & 76 & $13.7 \pm 1.7$ & $-1.62 \pm 1.3$ & $24.16 \pm 0.9$ & -0.90 \\
\hline 3) MH & 76 & $21.97 \pm 1.2$ & $-1.84 \pm 1.6$ & $24.21 \pm 0.8$ & -0.96 \\
\hline 4) MG & 84 & $15.9 \pm 2.6$ & $-1.95 \pm 1.55$ & $24.28 \pm 1.0$ & -0.88 \\
\hline & $\begin{array}{c}\text { Unpaired } \\
\text { T test }\end{array}$ & & $\mathrm{P}=0.38$ & $\mathrm{P}=0.72$ & $\mathrm{P}=0.0007$ \\
\hline
\end{tabular}

Refractive defects and axial length were similar between groups 2, 3 e 4 
3. GROUP 3: Myopes with ocular hypertension (MH). 17 males, 21 females, mean age $47.5 \pm 9.6$ years, mean IOP $21.97 \pm 1.2 \mathrm{mmHg}$, axial length $24.21 \pm 0.8 \mathrm{~mm}$, mean visual acuity $0.0078 \mathrm{LogMAR}$ (sd 0.2 ) with mean spherical equivalent $-1.84 \pm 1.6 \mathrm{D}, \mathrm{MD}-1.6 \mathrm{~dB}$ (sd 2.25), CS 1.78 LogMAR (sd 0.22), cup/disc ratio 0.2-0.4. Correlation CS-MD of $0.7895(\mathrm{P}<0.0001)$, correlation visual acuity-MD of $-0.4386(\mathrm{P}<0.0001)$.

4. GROUP 4: Myopes with primary open-angle glaucoma (MG). 19 males, 23 females, mean age $48 \pm 8.4$ years, mean IOP $15.9 \pm 2.6$ $\mathrm{mmHg}$ (patients in tonometric compensation under pharmacological therapy, with a mean disease duration of 5.38 years), mean visual acuity 0.06 LogMAR (sd 0.1) with mean spherical equivalent $-1.95 \pm$ 1.5 D, MD -5.032 dB (sd 6.36), CS 1.743 LogMAR (sd 0.19), cup/disc ratio 0.2-0.6. Correlation CS-MD of $0.8620(\mathrm{P}<0.0001)$, correlation visual acuity-MD of $-0.8921(\mathrm{P}<0.0001)$. (Table 2$)$

Contrast sensitivity (LogMAR): Our analysis showed that CS was reduced in the group of myopes with ocular hypertension and in the group of myopes with glaucoma, while it was preserved in the group of myopes and in the group of emmetropes. (Table 3,4)

Contrast sensitivity and automated visual field: correlating the results obtained on contrast sensitivity with those deriving from CSO vision chart (MD) we highlighted a statistically significant correlation between contrast sensitivity and mean deviation of the visual field H30-2. The greater the perimeter deficit, the proportionally smaller the contrast sensitivity. Our analysis showed that the myopic group differed by $31 \%$ from emmetropes and 53\% from hypertensive myopic (Table 5-7).

Visual acuity-mean deviation: the correlation between VA and $\mathrm{MD}$ is only present for myopic hypertensive patients and myopic glaucomatous groups and is significantly lower than the correlation between CS and MD (Table 8).

\section{Discussion}

Through our study we were able to evaluate how glaucomatous disease in myopic patients influences contrast sensitivity. Like other studies, our study too shows a reduction of contrast sensitivity in patients with glaucoma and in myopic patients with ocular hypertension.

Table 3. Contrast sensitivity (CS LogMAR) in the 4 groups

\begin{tabular}{|c|c|c|c|c|}
\hline GROUP & $\mathbf{N}$ & $\begin{array}{c}\text { CS } \\
\text { (LOGMAR) }\end{array}$ & SD & $\begin{array}{c}\text { BONFERRONI } \\
\text { TEST }\end{array}$ \\
\hline 1) $\mathrm{EM}$ & 80 & 2.069 & 0.16 & $\begin{array}{c}\text { NS VS } 2 \\
<0.001 \text { VS } 3,4\end{array}$ \\
\hline 2) $\mathrm{MY}$ & 76 & 1.990 & 0.17 & $\begin{array}{c}\text { NS VS } 1 \\
<0.001 \text { VS } 3,4\end{array}$ \\
\hline 3) $\mathrm{MH}$ & 76 & 1.788 & 0.22 & $\begin{array}{c}\text { NS VS } 4 \\
<0.001 \text { VS } 1,2\end{array}$ \\
\hline 4) $\mathrm{MG}$ & 84 & 1.743 & 0.19 & $\begin{array}{c}\text { NS VS } 3 \\
<0.001 \text { VS } 1,2\end{array}$ \\
\hline
\end{tabular}

One-way ANOVA $\mathrm{P}<0.00018$

Table 4. Contrast sensitivity in the 4 groups

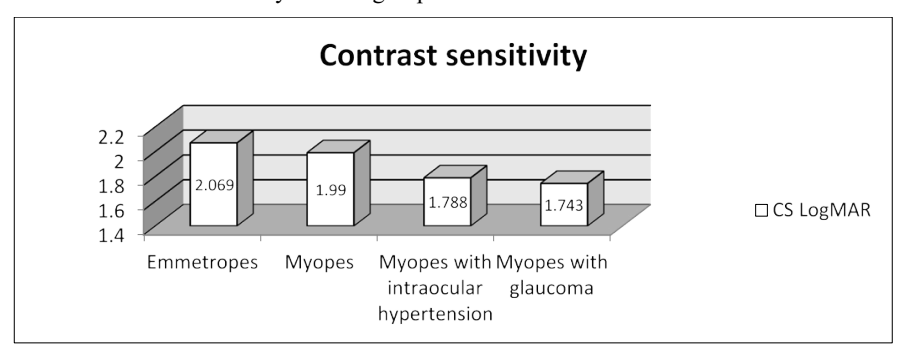

Table 5. Correlation between contrast sensitivity and visual field MD

\begin{tabular}{|l|c|c|c|c|c|}
\hline GROUP & N & MD (dB) & $\begin{array}{c}\text { Correlation(r) } \\
\text { CS-MD }\end{array}$ & $\begin{array}{c}\text { P value } \\
\text { CS-MD }\end{array}$ & P value \\
\hline 1) EM & 80 & 0.4563 & 0.3563 & $\mathrm{P}=0.0012$ & ES \\
\hline 2) MY & 76 & -0.098 & 0.5171 & $\mathrm{P}<0.0001$ & ES $31,4 \%$ \\
\hline 3) MH & 76 & -1.6 & 0.7895 & $\mathrm{P}<0.0001$ & ES $53 \%$ \\
\hline 4) MG & 84 & -5.032 & 0.8620 & $\mathrm{P}<0.0001$ & ES $10.2 \%$ \\
\hline
\end{tabular}

Table 6. Correlation between contrast sensitivity and visual field MD

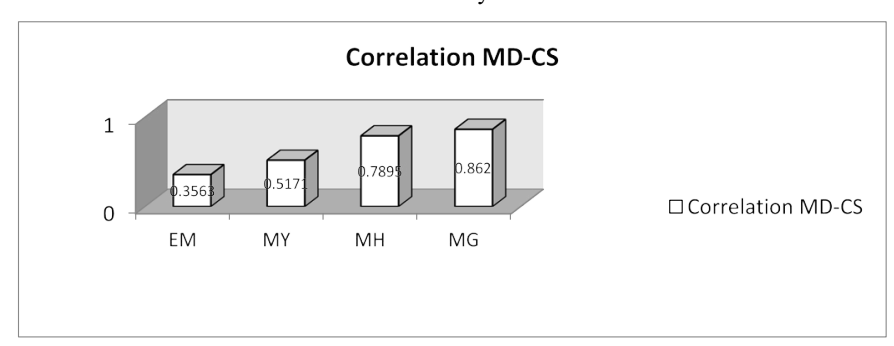

Table 7. Correlation between contrast sensitivity and visual field MD

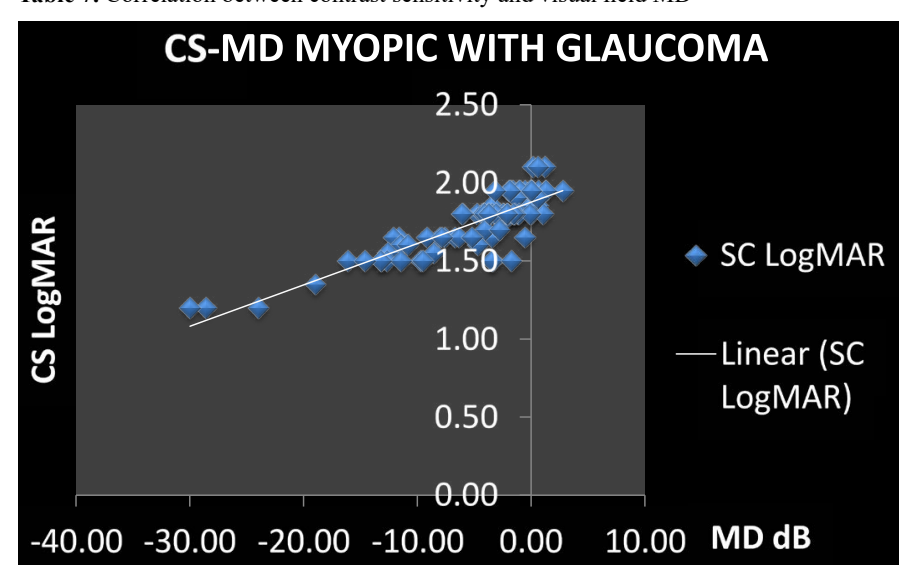

Table 8. Correlation between visual acuity and mean deviation

\begin{tabular}{|c|c|c|c|c|}
\hline GROUP & N & $\begin{array}{c}\text { Correlation (r) } \\
\text { VA-MD }\end{array}$ & $\begin{array}{c}\text { P value } \\
\text { VA-MD }\end{array}$ & P value \\
\hline 1) $\mathrm{EM}$ & 80 & -0.1753 & $\mathrm{P}=0.1198$ & $\mathrm{NS}$ \\
\hline 2) MY & 76 & -0.0265 & $\mathrm{P}=0.8198$ & $\mathrm{NS}$ \\
\hline 3) MH & 76 & -0.4386 & $\mathrm{P}<0.0001$ & $\mathrm{ES}$ \\
\hline 4) MG & 84 & -0.8921 & $\mathrm{P}<0.0001$ & $\mathrm{ES}$ \\
\hline
\end{tabular}

Our decision to evaluate contrast sensitivity in both myopic and myopic glaucomatous patients is linked to the fact that the two diseases are often found in association. According to the theory of Quigley [1], the optic nerve is subject to a stretching and traction effect of the lamina cribrosa lamellae due to scleral tension in the myopic eyes, much greater than that present in emmetropia [2], which could contribute to the development of glaucoma [13-16]. Moreover, myopia and glaucoma have a documented hereditary base, evidenced by the increased frequency of cortico-responders among the myopic subjects compared to the general population.

Our results confirm the loss of contrast sensitivity in myopic hypertensive and glaucomatous patients (1,788-1,743 LogMAR), but not in non-glaucomatous myopic patients (1,990 LogMAR).

In mild and medium grade myopia (up to 5 diopters) contrast sensitivity is conserved in the same way as emmetropes (2.069 LogMAR), contrary to what is shown by other studies in high-grade 
myopia. Thus, ocular hypertension seems to play an important role in reducing contrast sensitivity in patients with mild-to-medium myopia.

As already shown in other studies, our results also confirm a correlation between contrast sensitivity and the mean deviation of the visual field: the reduction of the retinal sensitivity of the visual field is accompanied by a directly proportional reduction of sensitivity to contrast. The choice of the most appropriate visual field parameter for this study fell on the mean deviation, because the damage induced by glaucoma on ganglion cells is widespread and not localized. The CSMD correlation is greater in patients with myopia and glaucoma $(\mathrm{r}=$ 0.86 ) and in medium grade myopic patients with ocular hypertension $(r=0.78)$; between the two groups there is only a $10 \%$ gap in percentile. As already described above, this may be related to the myopic eye anatomy which makes it more vulnerable to pressure increases and leads to greater damage to the optic nerve fibers.

It is also interesting to note that this correlation, the one between the retinal sensitivity assessed with the visual field and the contrast sensitivity, is opposed by a slight or zero correlation between visual acuity and perimetric damages: this relationship slightly subsists in the MH group and MG group, while it does not result significant in myopic and emmetropic patients.

\section{Conclusions}

We can conclude by stating that, from the data in our possession, it emerges how glaucomatous pathology in medium-myopic subjects involves a reduction not only of visual acuity, but also of the sensitivity to contrast in an important and early way; moreover, this reduction is correlated to the campimetric deficits to a greater extent than to visual acuity. This data is especially important in hypertensive patients, where the need for early diagnosis is necessary to decide whether to establish a therapy and therefore slow down and limit the progressive neuronal damage that glaucomatous pathology entails.

Therefore, from our study we could affirm that the Pelli-Robson test, fast and sensitive test even if not specific for glaucoma, should be performed in all patients, in particular in those with IOP at the upper limit of normal values range and optic papillae with aspects suspicious of glaucoma, given that the prevention and early diagnosis of glaucoma are still today the best defense against this disease.

\section{References}

1. Haegerstrom-Portnoy G (2005) The Glenn A. Fry Award Lecture 2003: Vision in elders--summary of findings of the SKI study. Optom Vis Sci 82: 87-93. [Crossref]

2. Owsley C (2003) Contrast sensitivity. Ophthalmol Clin North Am 16: 171-177. [Crossref]

3. Liu JL, McAnany JJ, Wilensky JT, Aref AA, Vajaranant TS (2017) "M\&S Smart System Contrast Sensitivity Measurements Compared with Standard Visual Function Measurements in Primary Open-Angle Glaucoma Patients". J Glaucoma 26: 528-533. [Crossref]

4. Hawkins AS, Szlyk JP, Ardickas Z, Alexander KR, Wilensky JT (2003) Comparison of contrast sensitivity, visual acuity, and Humphrey visual field testing in patients with glaucoma. J Glaucoma 12: 134-138. [Crossref]

5. Kerber KL, Thorn F, Bex PJ, Vera-Diaz FA (2016) Peripheral contrast sensitivity and attention in myopia. Vision Res 125: 49-54. [Crossref]

6. Jaworski A, Gentle A, Zele AJ, Vingrys AJ, McBrien NA (2006) Altered visua sensitivity in axial high myopia: a local postreceptoral phenomenon? Invest Ophthalmol Vis Sci 47: 3695-3702. [Crossref]

7. Arrico L, Giannotti R, Fratipietro M, Malagola R (2016) Fascicular Visual Field Defects in Open-Angle Glaucoma: Evaluation with Microperimetry. J Ophthalmol 2016: 8274954. [Crossref]

8. Arrico L, Nebbioso M, Komaiha C, Malagola R (2015) Utility of retinal thickness analyzer in early diagnosis of glaucomatous damage. In Vivo 29: 385-390. [Crossref]

9. Elliott DB, Sanderson K, Conkey A (1990) "The reliability of the Pelli-Robson contrast sensitivity chart”. Ophthalmic Physiol Opt 10: 21-24. [Crossref]

10. Haymes SA, Roberts KF, Cruess AF, Nicolela MT, LeBlanc RP (2006) "The Letter Contrast Sensitivity Test: clinical evaluation of a New Design" Invest. Ophthalmol Vis Sci 47: 2739-2745. [Crossref]

11. Stamper RL (1989) Psychophysical changes in glaucoma. Surv Ophthalmol 33 Suppl: 309-318. [Crossref]

12. Horn F, Martus P, Korth M (1993) "Comparison of temporal and spatiotemporal contrast-sensitivity tests in normal subjects and glaucoma patients". Ger J Ophthalmol 231: 146-150. [Crossref]

13. Sample PA, Juang PS, Weinreb RN (1991) Isolating the effects of primary openangle glaucoma on the contrast sensitivity function. Am J Ophthalmol 112: 308-316. [Crossref]

14. Quigley HA (1987) Reappraisal of the mechanisms of glaucomatous optic nerve damage. Eye (Lond) 1: 318-322. [Crossref]

15. Cahane M, Bartov E (1992) “Axial lenght and sclera thinckness effect on susceptibility to glaucomatous damage: a theorical model implementing Laplace's law". Ophtalmic Res 24: 22. [Crossref]

16. Perdicchi A, Arrico L, De Gregorio F, Pannarale L (1996) “Analysis of Visual Field Damage In Glaucomatous Myopic Eyes". Invest Ophthalmol Vis Sci 37: S513

Copyright: (C2018 Arrico L. This is an open-access article distributed under the terms of the Creative Commons Attribution License, which permits unrestricted use, distribution, and reproduction in any medium, provided the original author and source are credited. 